\title{
Produção de progesterona in vitro pelas células do corpo lúteo bovino ao longo da gestação ${ }^{1}$
}

\author{
Mariana N. La Paz ${ }^{2}$, Vanessa U. Fonseca², Danila B. Campos², Laura P. Artoni², \\ Liza M. M. C. Sousa ${ }^{2}$ e Paula C. Papa ${ }^{2 *}$
}

\begin{abstract}
La Paz M.N., Fonseca V.U., Campos D.B., Artoni L.P., Sousa L.M.M.C. \& Papa P.C. 2007. [In vitro progesterone production from bovine corpus luteum throughout gestation.] Produção de progesterona in vitro pelas células do corpo lúteo bovino ao longo da gestação. Pesquisa Veterinária Brasileira 27(9):370376. Setor de Anatomia, Departamento de Cirurgia, Faculdade de Medicina Veterinária e Zootecnia, Universidade de São Paulo, Av. Prof. Dr. Orlando Marques Paiva, 87, SP 05508-270, Brazil. E-mail: ppapa@usp.br

The aim was to test the hypothesis that cultivated bovine luteal cells from three different thirds of pregnancy behave the same way as in vivo luteal cells relative to $\mathrm{P} 4$ production. Corpus luteum samples from days $90(n=3), 150(n=3)$ and $210(n=3)$ of pregnancy were obtained at a local slaughterhouse. Under aseptic conditions cells were mechanically dispersed and cultivated in a 96 wells-plate. After 24 hours of culture, cells were washed and the precursor pregnenolone was added. Experiments were conducted eight times for each studied time period $(24,48$ and 96 h) and three times for each gestational age. Culture medium and cells were collected after 24, 48 and 96 hours of precursor addition and kept frozen at $-20^{\circ} \mathrm{C}$ until processing. Progesterone was measured by RIA and protein content by Lowry's method. Results were statistically analyzed and considered different when $\mathrm{p}<0.05$. A higher $\mathrm{P} 4$ production was observed on day 90 of gestation $(35.277 \pm 0.075)$, then this production was decreased at day $150(28.820 \pm 0.231)$ and increased again at day 210 (32.777 \pm 0.099$)$. After 24 hours of culture, luteal cells P4 production reached maximum values in the group of 90 days $(2.912 \pm 0.047)$ when compared to $150(2.669 \pm 0.137)$ and 210 days $(2.741 \pm 0.088)$. At 48 and 96 hours of culture, bovine luteal cells from day 90 of gestation produced more $\mathrm{P} 4$ than cells from day 210 ( $2.934 \pm 0.029$ and $2.976 \pm 0.121$ respectively x $2.760 \pm 0.059$ and $2.695 \pm 0.149$, respectively; $\mathrm{p}<0.05$ ), which in turn, produced more $\mathrm{P} 4$ than cells from day 150 ( $2.334 \pm 0.084$ for $48 \mathrm{~h}$ and $2.205 \pm 0.136$ for $96 \mathrm{~h}$ ). Luteal cells from day 150 of gestation presented a decreasing P4 production throughout the 96 hours of culture. These differences could be explained by differential gene expression of enzymes and/or factors belonging to the esteroidogenic cascade in accordance to the gestational period. The established luteal cell culture model could be used for further functional studies once P4 secretion pattern in vitro resembled what occurs in vivo.
\end{abstract}

INDEX TERMS: Corpus luteum, gestation, progesterone, cell culture.

RESUMO.- O presente trabalho foi desenvolvido para testar a hipótese de que células luteínicas bovinas em cultivo, provenientes dos três terços de gestação, comportam-se da mesma maneira que células in vivo em relação à produção de $\mathrm{P} 4$. Foram coletadas amostras de corpos lúteos $(\mathrm{CL})$ de $90(\mathrm{n}=3)$,

\footnotetext{
${ }^{1}$ Recebido em 16 de junho de 2007.

Aceito para publicação em 18 de setembro de 2007.

${ }^{2}$ Setor de Anatomia, Departamento de Cirurgia, Faculdade de Medicina Veterinária e Zootecnia, Universidade de São Paulo, Av. Prof. Dr. Orlando Marques Paiva 87, SP 05508-270, Brazil. *E-mail: ppapa@usp.br
}

$150(n=3)$ e $210(n=3)$ dias de gestação obtidos em abatedouro. Sob condições assépticas, as células foram mecanicamente dispersas e cultivadas em placas de 96 poços. Após 24 horas de cultivo foram feitas a lavagem dos poços e a adição do precursor pregnenolona. Os tratamentos foram realizados em octuplicata para cada tempo de tratamento $(24$, 48 e 96 horas) com três repetições de cada período gestacional. As amostras de meio de cultura e as células foram coletadas 24, 48 e 96 horas após adição do precursor e acondicionadas em freezer a $-20^{\circ} \mathrm{C}$ até o processamento. A progesterona foi dosada através de radioimunoensaio e o 
conteúdo protéico pelo método de Lowry. Os resultados foram analisados estatisticamente e considerados diferentes quando $\mathrm{p}<0.05$. Foi observada maior produção de $\mathrm{P} 4$ aos 90 dias de gestação $(35,277 \pm 0,075)$, posterior decréscimo aos 150 dias $(28,820 \pm 0,231)$ e novo aumento aos 210 dias $(32,777 \pm 0,099)$. A produção de $\mathrm{P} 4$ em células cultivadas por 24 horas foi maior $(p<0,05)$ em células oriundas do grupo de 90 dias $(2,912 \pm 0,047)$ quando comparado a $150(2,669 \pm 0,137)$ e 210 dias $(2,741 \pm 0,088)$. As 48 e 96 horas de cultivo, células luteínicas bovinas de 90 dias produziram mais $\mathrm{P} 4$ que células de 210 dias $(2,934 \pm 0,029$ e $2,976 \pm 0,121$ respectivamente $x$ $2,760 \pm 0,059$ e $2,695 \pm 0,149$, respectivamente; $p<0,05$ ), que por sua vez produziram mais do que células de 150 dias $(2,334 \pm 0,084$ para 48 horas e $2,205 \pm 0,136$ para 96 horas). Aos 150 dias de gestação a produção de progesterona apresentou diminuição gradativa ao longo das 96 horas de cultivo. Essas diferenças podem ser explicadas pela expressão gênica diferencial de enzimas ou também de fatores presentes na cascata esteroidogênica de acordo com a idade gestacional. Este modelo de cultura celular luteínica poderá ser utilizado em estudos funcionais uma vez que o padrão de secreção de P4 mimetizou o que ocorre in vivo.

TERMOS DE INDEXAÇÃO: Corpo lúteo, gestação, progesterona, cultivo celular.

\section{INTRODUÇÃO}

O corpo lúteo $(\mathrm{CL})$ é uma glândula reprodutiva, transitória, produtora de progesterona (P4), necessária ao estabelecimento e manutenção da prenhez (Schams \& Berisha 2004). Este hormônio é responsável por manter um ambiente uterino ideal para o desenvolvimento, bem como a receptividade uterina ao concepto e a secreção endometrial necessária para o desenvolvimento inicial do embrião e adesão placentária, além de regular a contratilidade miometrial (Geisert \& Conley 1998).

A regulação da função luteínica é feita por um complexo grupo de agentes que interagem na busca da homeostasia da glândula. Os fatores de crescimento, principalmente o VEGF (vascular endothelial growth factor) e o bFGF (basic fibroblastic growth factor), são responsáveis pelo desenvolvimento e manutenção da densa rede de capilares neo-formados, além de contribuírem de maneira parácrina e autócrina para a produção de P4 (Schams \& Berisha 2002, Campos 2005, Needle et al. 2007). Secundariamente, o hormônio luteinizante (LH) e o hormônio do crescimento $(\mathrm{GH})$, possuem uma ação efetiva na regulação da atuação dos fatores de crescimento e estímulo à produção de $\mathrm{P} 4$; outros agentes, como peptídeos, esteróides e prostaglandinas são responsáveis pela modulação da função luteínica e regulação fina da glândula (Schams \& Berisha 2004).

A produção de progesterona pelo CL ocorre a partir da pregnenolona, um precursor esteróide formado pela quebra da cadeia lateral do colesterol, mediante estimulação hormonal de LH, angiotensina II e hormônio corticotrófico (Kufe et al. 2003). Durante o ciclo estral da fêmea bovina, o LH é secretado em baixos níveis com aumento da amplitude, exceto durante a onda pré-ovulatória. Estes estímulos em conjunto promovem o ingurgitamento do folículo, a ovulação, e permitem a formação do CL (Ayad et al. 2007) a partir das células remanescentes do folículo: células da teca e da granulosa, que apresentam alta capacidade proliferativa (Denschlag \& Keck 2002). As células da teca se diferenciam em células luteínicas pequenas (SLC) e as da granulosa em células luteínicas grandes (LLC); apenas as SLC apresentam receptores para LH e, portanto a capacidade de captar colesterol (Farin et al. 1989).

Segundo Niswender et al. (1994) e Milvae et al. (1996), o mecanismo pelo qual o LH estimula a secreção de P4 nas células luteínicas pequenas (SLC) envolve a formação de cAMP e a ativação do sistema da proteína quinase $\mathrm{A}$, que promove a fosforilação das enzimas envolvidas na esteroidogênese e maximiza o transporte de colesterol para o citoplasma, especialmente para o interior da membrana mitocondrial das pequenas células luteínicas. Após o transporte de colesterol para a matriz mitocondrial, ocorre a clivagem da cadeia lateral, produzindo assim a pregnenolona, que em seguida é convertida em P4.

Na vaca não prenhe, a prostaglandina $F_{2 \alpha}$ endometrial $\left(\mathrm{PGF}_{2 \alpha}\right)$ é capaz de dar início ao processo luteolítico entre o $15^{\circ}$ e $17^{\circ}$ dias após a ovulação (Northey \& French 1980). Caso contrário, na presença de um concepto viável, a secreção de proteínas trofoblásticas impede a liberação de $\mathrm{PGF}_{2 \alpha}$ pelo endométrio (Bazer et al. 1986, Godkin et al. 1988) e facilita a manutenção do CL (Knickerbocker et al. 1986). Nos bovinos o IFN- $\tau$ (interferon tau) é uma glicoproteína secretada pelo trofoblasto, que atua como uma molécula anti-luteolítica. Ele é sintetizado por células mononucleares do trofoectoderma, e exclusivamente secretado no lúmen uterino (Roberts et al. 1992, Morgan et al. 1993). Enquanto um aumento na concentração de mRNA do interferon- $\tau$ pode ser detectada entre $o$ $9^{\circ}$ e $10^{\circ}$ dias de gestação (Kubisch et al. 2001), a produção em larga escala só é observada entre os dias 17 e 19 pós-ovulação, quando o concepto sofre rápida expansão em sua forma alongada (Farin et al. 1990).

Um grande número de estudos mostrou que a alta concentração de $\mathrm{P} 4$ poderia suprir adequadamente o ambiente uterino para o desenvolvimento embrionário, resultando em um concepto grande que é capaz de produzir mais proteínas trofoblásticas (Kerbler et al. 1997, Mann et al. 2006). Outras famílias de glicoproteínas do trofoblasto também são expressas pelo concepto bovino, ao mesmo tempo ou anteriores a expressão do interferon- $\tau$ (Ushizawa et al. 2004). Entre elas estão as glicoproteínas associada à gestação (PAGs), a proteína específica da prenhez (PSPB) ou proteína soro-prenhez 60kDa (PSP60, Xie et al. 1994, 1997, Green et al. 2000, Garbayo et al. 2007).

As altas concentrações de P4 luteínica se mantêm até o $180^{\circ}$ dia de gestação, declinando gradativamente (Shemesh et al. 1984); a partir deste momento, a placenta que até então havia apresentado níveis oscilantes de produção, passa a produzir o hormônio em maior escala (Shemesh et al. 1992, 1994).

Devido à grande importância do estudo da esteroidogênese luteínica gestacional e do estabelecimento de modelos de estudo para este fim, testou-se a hipótese de que o com- 
portamento in vitro das células luteínicas gestacionais bovinas provenientes dos três diferentes terços de gestação obedece ao mesmo padrão para a secreção de progesterona observado in vivo.

\section{MATERIAL E MÉTODOS}

Corpos lúteos bovinos de $90(n=3), 150(n=3)$ e 210 dias $(n=3)$ de gestação, correspondentes aos primeiro, segundo e terceiro terços gestacionais, respectivamente, obtidos em abatedouro, foram lavados em solução isotônica estéril de cloreto de sódio $0,9 \%$ e transportados ao laboratório a $4^{\circ} \mathrm{C}$ em PBS contendo penicilina, estreptomicina e anfotericina-B (Sigma, A5955). Para a determinação da idade gestacional foi mensurado o comprimento cefalococcígeo do feto ("Crown Rump"), de acordo com Sloss \& Dufty (1980).

O cultivo celular foi estabelecido com base na metodologia descrita por Grasselli et al. (2002) para células da granulosa. Resumidamente: sob condições assépticas, os CL foram cortados em fragmentos de $0,5 \mathrm{~cm}^{3}$ e as células foram mecanicamente separadas pela passagem através de uma rede de 150 mesh acoplada a um Becker coletor e em seguida, lavadas e centrifugadas três vezes em meio de cultura D-MEM (Sigma, D5523) suplementado com 5\% de soro fetal bovino (Gibco Life, $10270-106$ ) inativado a $56^{\circ} \mathrm{C}$ por 30 minutos, $2 \%$ de L-Glutamina 3\% (Sigma, G8540), 5\% de HEPES $280 \mathrm{mM}$ (Sigma, H4034), 0,5\% de bicarbonato de sódio 7,5\% (Amresco, 0865) e solução de antibiótico e antifúngico (100.000 UI de penicilina, $100 \mathrm{mg}$ de estreptomicina e 250 ìg de anfotericina-B, Sigma, A5955).

As células foram cultivadas em placas de 96 poços $(0,64 \mathrm{~cm}$ diâmetro x 0,96cm altura) (Costar ${ }^{\circledR}$, Corning, USA) em incubadora umidificada, com uma atmosfera de $5 \%$ de $\mathrm{CO}_{2}$ e temperatura de $37,5^{\circ} \mathrm{C}$. Foi utilizada uma concentração de $5 \times 10^{6}$ células viáveis em $300 \mu l$ de meio de cultivo em cada poço. Após 24 horas de cultivo para adaptação das células, foi feita lavagem e adição do precursor pregnenolona (P5) na concentração de 0,003g/ml (Sigma, P9129) em oito poços diferentes para cada tempo de tratamento.

As amostras foram coletadas 24,48 e 96 horas após a adição da P5. Coletado o meio de cultura, os poços foram tripsinizados, através de pipetagens/lavagens e ressuspensões, com $0.25 \mathrm{mg} / \mathrm{ml}$ de tripsina suína (Sigma, T4549), incubados em estufa a $37,5^{\circ} \mathrm{C}$ e o conteúdo protéico foi mensurado pelo Método de Lowry modificado (Lowry et al. 1951). A concentração de progesterona foi mensurada por radioimunoensaio (RIA) usando o kit comercial específico (DSL-3400). Para cálculo da concentração de progesterona por idade gestacional e/ou tempo de tratamento, a concentração medida no meio (ng/ml) foi corrigida pelo conteúdo protéico $(\mathrm{mg} / \mathrm{ml})$ presente em seu respectivo poço. $\mathrm{O}$ efeito do tempo de cultivo e da idade gestacional sobre a concentração de progesterona por conteúdo protéico nos poços foram analisados pelo programa estatístico Minitab ${ }^{\circledR}$. Quando a distribuição dos dados foi considerada normal e as variâncias iguais, os dados foram analisados por ANOVA. Os dados que apresentaram distribuição não paramétrica foram analisados por Kruskal-Wallis. As médias foram consideradas diferentes quando $\mathrm{p}<0,05$.

\section{RESULTADOS}

O presente estudo avaliou o comportamento in vitro das células luteínicas quanto à produção de progesterona em três diferentes fases da gestação. Foram encontradas modificações significativas no perfil de secreção de progesterona pelas células luteínicas durante os três períodos gestacionais analisados.

Foi observada maior $(\mathrm{p}<0,05)$ produção total de proges-

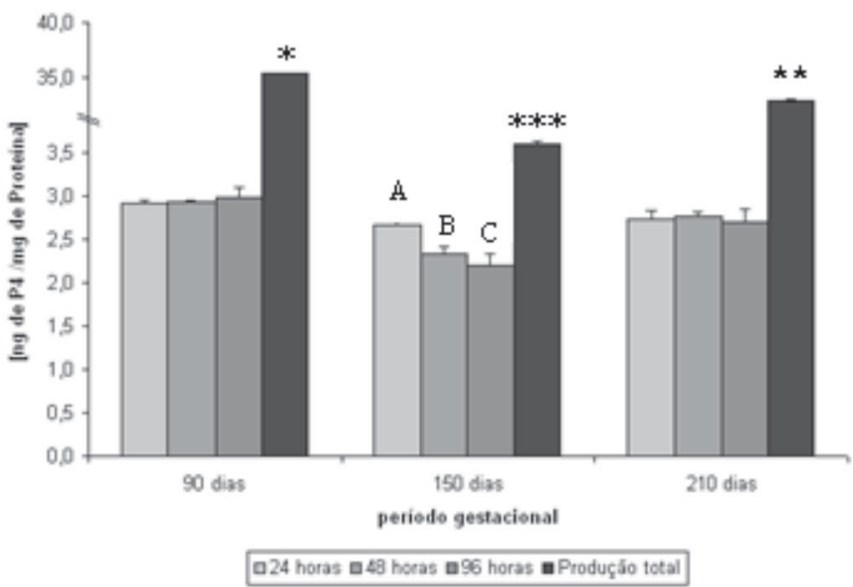

Fig.1. Médias ( \pm desvios padrões) das concentrações in vitro de Progesterona, por período de gestação e total, produzida pelas células do corpo lúteo aos 90, 150 e 210 dias de gestação, em amostras coletadas 24, 48 e 96 horas após a adição do precursor pregnenolona (Sigma, P9129). Letras e $\mathbf{n}^{-}$de asteriscos diferentes diferem significativamente $(\mathrm{P}<0,05)$.

terona in vitro aos 90 dias de gestação $(35,277 \pm 0,075)$ comparativamente aos demais períodos gestacionais $(28,820 \pm$ 0,231 e 32,777 0,099; para 150 e 210 dias respectivamente, Quadro 1). Aos 150 dias esta produção decresceu $(p<0,05)$ e aos 210 dias aumentou novamente ( $<<0,05$; Fig.1).

$O$ perfil de adaptação das células em cultura, de acordo com o terço gestacional estudado, foi analisado a partir da mensuração da produção de $\mathrm{P} 4$ as 24,48 e 96 horas de cultivo (Quadro 1). Observou-se que as 24 horas houve uma produção significativamente maior aos 90 dias $(2,912 \pm 0,047$; $\mathrm{p}<0,05)$ em relação aos 150 dias $(2,668 \pm 0,137)$. Aos 210 dias de gestação, a produção de $\mathrm{P} 4$ as 24 horas de cultivo apresentou valores intermediários em relação aos outros dois períodos (2,741 $\pm 0,088$; Fig.2). As 48 horas de cultivo verificou-se uma produção significativamente maior $(\mathrm{p}<0,05)$ de $\mathrm{P} 4$ aos 90 dias de gestação $(2,9340,029)$ em relação aos 210 dias $(2,760 \pm 0,059)$, e este último em relação aos 150 dias $(2,334 \pm 0,084)$. 0 perfil de produção às 96 horas de cultivo seguiu o mesmo padrão encontrado para 48 horas de cultivo (Fig.2).

Quadro 1. Concentração de progesterona $(\eta \mathrm{g} / \mathrm{ml})$ por período gestacional de acordo com o tempo de cultivo e concentração total de progesterona $(\eta \mathrm{g} / \mathrm{ml})$ no meio de cultura de células luteínicas bovinas em diferentes períodos gestacionais

\begin{tabular}{cccc}
\hline Tempo de cultivo & \multicolumn{3}{c}{ Período gestacional (dias) } \\
\cline { 2 - 4 } & 90 & 150 & 210 \\
\hline 24 horas & $2,912 \pm 0,047^{\mathrm{a}}$ & $2,668 \pm 0,137^{\mathrm{bA}}$ & $2,741 \pm 0,088^{\mathrm{a}, \mathrm{b}}$ \\
48 horas & $2,934 \pm 0,029^{\mathrm{a}}$ & $2,334 \pm 0,084^{\mathrm{cB}}$ & $2,760 \pm 0,059^{\mathrm{b}}$ \\
96 horas & $2,976 \pm 0,121^{\mathrm{a}}$ & $2,205 \pm 0,136^{\mathrm{cC}}$ & $2,695 \pm 0,149^{\mathrm{b}}$ \\
Concentração total & $35,277 \pm 0,075^{\mathrm{a}}$ & $28,820 \pm 0,231^{\mathrm{c}}$ & $32,777 \pm 0,099^{\mathrm{b}}$
\end{tabular}

Valores representam a média \pm DP de três diferentes experimentos para cada período gestacional $(\mathrm{n}=24$ por tempo de tratamento) e a média $\pm \mathrm{DP}$ de todos os valores obtidos para cada período gestacional $(\mathrm{n}=72$ por período). Letras diferentes: diferenças significativas $(\mathrm{P}<0.05)$ entre o tempo de cultivo $(A, B, C)$ ou entre o período gestacional estudado $(a, b, c)$. 


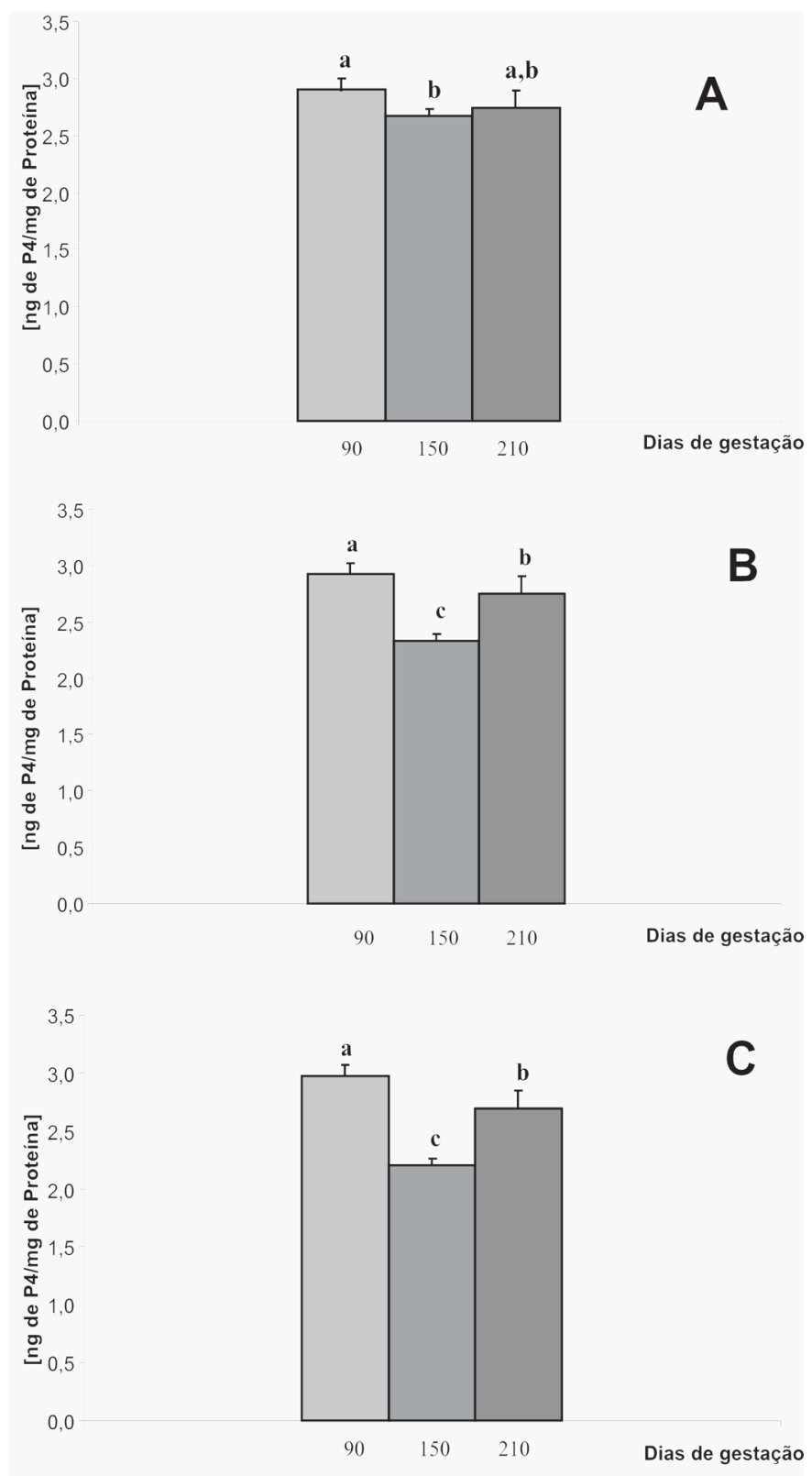

Fig.2. Médias ( \pm desvios padrões) das concentrações in vitro de Progesterona por tempo de tratamento: 24 horas (A), 48 horas (B) e 96 horas (C), produzida pelas células do corpo lúteo aos 90 , 150 e 210 dias de gestação, em amostras coletadas após a adição do precursor pregnenolona (Sigma, P9129). Letras diferentes diferem significativamente $(\mathrm{P}<0,05)$.

Quando as células dos diferentes terços gestacionais foram analisadas separadamente em relação ao perfil de produção de $\mathrm{P} 4$ durante as 96 horas de cultivo, observou-se que células provenientes do primeiro e terceiro terços gestacionais, $90(35,277 \pm 0,075)$ e 210 dias $(32,777 \pm 0,099)$ produzem a mesma quantidade de $\mathrm{P} 4$ desde 24 até 96 horas de cultivo (Fig. 1, Quadro1). Células luteínicas de 150 dias de gestação apresentam produção decrescente $(\mathrm{p}<0,05)$ de progesterona ao longo do período estudado (24>48>96 horas, Fig.1).

\section{DISCUSSÃO E CONCLUSÃO}

Em mamíferos, a progesterona (P4) é de suma importância para o estabelecimento e manutenção de um ambiente uterino adequado para o desenvolvimento embrionário e fetal. Nos bovinos, a habilidade do corpo lúteo cíclico de produzir progesterona tanto in vivo quanto in vitro já está bem estabelecida: a síntese e concentrações de P4 apresentam variações cíclicas, as quais refletem a funcionalidade do corpo lúteo ao longo das diferentes fases de crescimento, manutenção e regressão (Milvae \& Hansel 1983, Kobayashi et al. 2001, Wolfenson et al. 2002, Nicklin et al. 2007). No presente estudo, células provenientes de corpos lúteos de diferentes terços gestacionais ( 90 dias, primeiro terço, 150 dias, segundo terço e 210 dias, terceiro terço) foram cultivadas sob as mesmas condições e apresentaram diferentes $(p<0,05)$ quantidades de produção de progesterona. A secreção de P4 luteínica no meio de cultivo apresentou tendência a aumento no terço inicial (90 dias; Fig.1), após 48 e 96 horas de experimento (Fig.2) sugerindo efeito cumulativo do hormônio no meio de cultivo ao longo do tempo e maior atividade celular após um período de adaptação ao ambiente e condições de cultura. Tal fato foi também observado por Campos (2005) ao analisar a produção de $\mathrm{P} 4$ in vitro em células placentárias bovinas. A maior secreção de P4 no início da gestação é condizente com os achados de Shaha \& Greenwald (1982), Yuan et al. (1993) e Ayad et al. (2007) ao estudarem a síntese hormonal in vitro em CL gestacionais de hamsters, suínos e bovinos, respectivamente. A progesterona luteínica atua no endométrio modulando a regulação de genes importantes para a receptividade uterina e crescimento do concepto durante a fase inicial da gestação (Ayad et al. 2007).

À medida que avança a gestação, a atividade secretória do CL diminui gradativamente devido a ação da PGF $_{2 \alpha}$ (Baird 1992). Um pico inicial de prostaglandina de origem endometrial, mesmo de baixa magnitude, pode potencialmente iniciar os mecanismos para que as grandes células luteínicas produzam consideráveis concentrações locais de $\operatorname{PGF}_{2 \alpha}$ (Tsai $\&$ Wiltbank 1997). O presente trabalho demonstrou uma queda significativa na secreção de P4 aos 150 dias (Fig.1), não somente em relação aos 90 dias como também em função do tempo de incubação, atingindo menor concentração no período de 96 horas (Fig.2). Segundo Milvae et al. (1996), o decréscimo na produção de progesterona deve-se mais comumente à diminuição da capacidade esteroidogênica das células luteínicas individualmente. A regressão morfológica, focada atualmente no estudo dos processos apoptóticos, sugere que as células não esteroidogênicas (células do estroma luteínico e fibroblastos) presentes no CL sofram apoptose, possivelmente iniciada por hipóxia (Niswender et al. 1990), explicando a queda de produção hormonal nessa fase pelas células cultivadas. Por outro lado, o aumento na síntese de P4 observado nas células luteínicas aos 210 dias (Fig.1) não era esperado, uma vez que evidências para a deterioração morfológica do CL bovino são observadas mais precocemente a partir do dia 200 de gestação, momento este em que a placenta assume o papel de fonte de progesterona (Fields \& Fields 1996). Por outro lado, aos 210 dias de gestação o CL 
apresenta expressão máxima do mRNA do VEGF (Garbelotti 2006), o que pode contribuir para aumento da síntese de $P 4$ nesta fase (Papa et al. 2006, Needle et al. 2007). No corpo lúteo cíclico de búfalos foi observada alta correlação existente entre a produção de progesterona e a expressão de mRNA e proteína do VEGF (Papa et al. 2006) bem como entre produção de P4 e densidade vascular (Moura et al. 2003).

O substrato para a síntese de progesterona é o colesterol, que precisa ser transportado para o interior da membrana mitocondrial. Este transporte requer um citoesqueleto intacto e a fosforilação da proteína esteroidogênica regulatória aguda (StAR). Uma vez alcançada a matriz mitocondrial, a enzima citocromo P450scc (cholesterol side chain cleavage cytochrome P450) atua na conversão do colesterol em pregnenolona (P5) que é transportada para o retículo endoplasmático liso (REL), no qual a enzima $3 \alpha$-hidroxiesteróide dehidrogenase/ $\Delta_{4}, \Delta_{5}$ isomerase $(3 \alpha-\mathrm{HSD})$ converte a pregnenolona em progesterona, esta última então rapidamente secretada (Niswender et al. 2000, Niswender 2002). Em bovinos (Voss \& Fortune, 1993), assim como nos ovinos (Juengel et al. 1994) e suínos (Conley et al. 1992), a ovulação está associada a uma diminuição da expressão de enzimas que convertem progesterona a estrógenos, isto é, a $17 \alpha$-hidroxilase/C17, 20-liase (P450c17 $\alpha$ ) e a aromatase $\mathrm{P} 450$, e um concomitante aumento na expressão da enzima $3 \beta$-HSD no parênquima do corpo lúteo em desenvolvimento. Em suínos, porém, algumas células que expressam a $\mathrm{P} 450 \mathrm{c} 17 \alpha$ permanecem e se dispersam por todo o parênquima do CL maduro, o que explica a habilidade das células luteínicas suínas em sintetizar androstenediona (Pitzel et al. 1988) e estrógenos (Einspanier et al. 1991). No entanto, no corpo lúteo bovino em desenvolvimento, há um aumento da proporção de células que expressam a enzima $3 \beta-H S D$ e a fase de regressão do corpo lúteo é caracterizada pelo declínio no número de células parenquimais que expressam esta enzima. Com base nos dados acima descritos, pode-se sugerir que a regulação da expressão da enzima $3 \beta$-HSD também possa ocorrer no corpo lúteo gestacional e determine a aumento e/ou diminuição da secreção de progesterona (Conley et al. 1995, Bao \& Garverick 1998).

O comportamento celular luteínico para produção de P4 ao longo do tempo de cultivo também apresentou variações dependentes do estágio gestacional estudado (Fig.2), o que sugere uma modulação fina dos mecanismos regulatórios desta produção (Schams \& Berisha 2002). O CL cíclico nos bovinos é uma estrutura dinâmica com um período de vida de aproximadamente de 17-18 dias (Ohtani et al. 1998). A concentração de P4 é baixa no dia 0 (dia do estro), seguindo um perfil ascendente até aproximadamente o $14^{\circ}$ dia, com valores médios que atingem um máximo de $5,9 \mathrm{ng} / \mathrm{ml}$ (Borges et al. 2003). Tal aumento ocorre devido à organização do tecido luteínico, com concomitante aumento do fluxo sanguíneo ovariano e peso do CL (Wiltbank et al. 1995). Na ausência da prenhez, a $\mathrm{PGF}_{2 \alpha}$ inicia o processo luteolítico entre os dias 15 e 17 após a ovulação (Northey \& French 1980), no qual o CL é submetido à regressão morfológica e funcional (Milvae et al. 2000), caracterizada pela queda da produção de progesterona e perda dos componentes celulares, incluindo redução do suprimento vascular, proliferação do tecido conjuntivo, aumento da desorganização celular, degeneração e fagocitose das células luteínicas (Carlson et al. 1982, Pate 1994, Miyamoto 1996). Por outro lado, na presença de um concepto viável, a secreção de proteínas tais como o IFN- $\tau$ previne a liberação de pulsos de $\mathrm{PGF}_{2 \alpha}$ pelo endométrio, facilitando a manutenção do CL e conseqüientemente a produção de $\mathrm{P} 4$ até o terço final da gestação (Fields \& Fields 1996) o que corrobora com os dados do presente trabalho.

Assim, o presente trabalho demonstrou que as células de corpo lúteo gestacional bovino em cultivo secretam progesterona preferencialmente durante a fase inicial da gestação, seguindo o perfil esteroidogênico descrito para a espécie e que o comportamento desta produção ao longo do cultivo é dependente da fase gestacional. Este modelo de cultivo celular luteínico poderá vir a ser utilizado em estudos funcionais.

Agradecimentos.- À FAPESP (Proc. 02/07392-7, 05/50243-0 e 05/51899-7), pelo financiamento desta pesquisa.

\section{REFERÊNCIAS}

Ayad A., Sousa N.M., Sulon J., Hornick J.L., Watts J., Lopez-Gatius F., IguerOuada M. \& Beckers J.F. 2007. Influence of progesterone concentrations on secretory functions of trophoblast and pituitary during the first trimester of pregnancy in dairy cattle. Theriogenology 67:1503-1511.

Baird D.T. 1992. Luteotropic control of the corpus luteum. Anim. Reprod. Sci. 28:95-102.

Bao B. \& Garverick H.A. 1998. Expression of steroidogenic enzyme and gonadotropin receptor genes in bovine follicles during ovarian follicular waves: a review. J. Anim. Sci. 76:1903-1921.

Bazer F.W., Vallet J.L., Roberts R.M., Sharp D.C. \& Thatcher W.W. 1986. Role of conceptus secretory products in establishment of pregnancy. J. Reprod. Fertil. 76:841-50.

Borges A.M., Torres C.A.A., Ruas J.R.M., Rocha JR. V.R. \& Carvalho G.R. 2003. Desenvolvimento luteal e concentrações plasmáticas de progesterona em vacas das raças Gir e Nelore. Revta Bras. Zootec. 32:276-283.

Campos D.B. 2005. Imunolocalização do VEGF, bFGF e seus receptores na placenta bovina e influência destes fatores sobre a produção de progesterona pelas células placentárias em cultura. Tese de Doutorado em Ciências, Faculdade de Medicina Veterinária e Zootecnia, Universidade de São Paulo, São Paulo. 193p.

Carlson J.A., Buhr M., Wentworth R. \& Hansel W. 1982. Evidence of membrane changes during regression in the bovine corpus luteum. Endocrinology 110:1472-1476.

Conley A.J., Kaminski M.A, Dubowsky S.A., Jablonla-Shariff A., Redmer D.A. \& Reynolds L.P. 1995. Immunohistochemical localization of 3 betahydroxysteroid dehydrogenase and P450 17 alpha-hydroxylase during follicular and luteal development in pigs, sheep, and cows. Biol. Reprod. 52:1081-1094.

Denschlag D. \& Keck C. 2002. The Corpus luteum. Tierärztl. Umschau 59(4):159162.

Einspanier A., Jarry H., Pitzel L., Holtz W. \& Wuttke W. 1991. Determination of secretion rates of estradiol, progesterone, oxytocin, and angiotensin II from tertiary follicles and freshly formed corpora lutea in freely moving sows. Endocrinology 129:3403-3409.

Fields M.J. \& Fields P.A. 1996. Morphlogical characteristics of the bovine corpus luteum during the estrous and pregnancy. Theriogenology 45:12951325.

Farin C.E., Sawyer H.R. \& Niswender G.D. 1989. Analysis of cell types in the corpus luteum of the sheep. J. Reprod. Fertil. 37(Suppl.):181-187.

Farin C.E., Imakawa K., Hansen T.R., McDonnell J.J., Murphy C.N., Farin P.W., 
Imakaua K \& Hansen TR. 1990. Expression of trophoblastic interferon genes in sheep and cattle. Biol Reprod. 43:210-218.

Garbayo J.M., Serrano B. \& Lopez-Gatius F. 2007. Identification of novel pregnancy-associated glycoproteins (PAG) expressed by the periimplantation conceptus of domestic ruminants. Anim. Reprod. Sci. doi:10.1016/j.anireprosci.2006.12.002.

Garbelotti F. 2006. Expressão do mRNA do VEGF, Flt-1 e KDR no placentoma, região interplacentomal e corpo lúteo em diferentes fases gestacionais em bovinos clonados e não clonados. Dissertação de Mestrado em Ciências, Faculdade de Medicina Veterinária e Zootecnia, Universidade de São Paulo, São Paulo. 110p.

Geisert R.D. \& Conley A.J. 1998. Secretion and metabolism of steroids in subprimate mammals during pregnancy, p.291-318. In: Bazer F.W. (ed.), Contemporany Endocrinology. Vol.9, The Endocrinology of Pregnancy. Human Press Inc.,Totowa.

Godkin J.D., Lifsey Jr. B.J. \& Gillespie B.E. 1988. Characterization of bovine conceptus proteins produced during the peri- and postattachment periods of early pregnancy. Biol Reprod. 38:703-711.

Grasselli F., Basini G., Bussolati S. \& Tamanini C. 2002. Effects of VEGF and bFGF on proliferation and production of steroids and nitric oxide in porcine granulosa cells. Reprod. Domest. Anim. 37(6):362-368.

Green J.A., Xie S., Quan X., Bao B., Gan X., Mathialagan N., Beckers J.F. \& Roberts R.M. 2000. Pregnancy-associated bovine and ovine glycoproteins exhibit spatially and temporally distinct expression patterns during pregnancy. Biol. Reprod. 62:1624-1631.

Hawkins D.E., Belfiore C.J., Kile J.P. \& Niswender G.D. 1993. Regulation of messenger ribonucleic acid encoding 3-hydroxysteroid dehydrogenase/A 5-A4-isomerase in the ovine corpus luteum. Biol. Reprod. 48:1185-1190.

Jeungel J.L., Guy M.K., Tandeski T.R., McGuire W.J. \& Niswender G.D. 1994. Steady-state concentrations of messenger ribonucleic acid encoding cytochrome P450 cholesterol side-chain cleavage and 3-hydroxysteroid dehydrogenase/A5 , A4 isomerase in ovine corpora lutea during the estrous cycle. Biol. Reprod. 51:380-384.

Kerbler T.L., Buhr M.M., Jordan L.T., Leslie K.E. \& Walton J.S. 1997. Relationship between maternal plasma progesterone concentration and interferon-tau synthesis by the conceptus in cattle. Theriogenology 47:703-714.

Knickerbocker J.J., Thatcher W.W., Bazer F.W., Drost M., Barron D.H., Fincher K.B. \& Roberts R.M. 1986. Proteins secreted by day-16 to -18 bovine conceptuses extend corpus luteum function in cows. J. Reprod. Fertil. 77:381-391

Kobayashi S., Berisha B., Amselgruber W.M., Schams D. \& Miyamoto A. 2001. Production and localization of angiotensin II in the bovine early corpus luteum: a possible interaction with luteal angiogenic factors and prostaglandin F2?. J. Endocrinology 170:369-380.

Kubisch H.M., Larson M.A., Ealy A.D., Murphy C.N. \& Roberts R.M. 2001. Genetic and environmental determinants of interferon-tau secretion by in vivo- and in vitro-derived bovine blastocysts. Anim. Reprod. Sci. 66:113.

Kufe D.W., Pollock R.E., Weichselbaum R.R., Bast Jr R.C.,Gansler T.S., Holland J.F. \& Frei lii E. 2003. Cancer Medicine. 6th ed. B.C. Decker Inc., Hamilton, Ontario, Canada, p.911-926.

Lowry O.H., Rosebrough N.J., Farr A.L. \& Randall R.J. 1951. Protein measurement with the Folin phenol reagent. J. Biol. Chem. 193:265-275.

Mann G.E., Fray M.D. \& Lamming G.E. 2006. Effects of time of progesterone supplementation on embryo development and interferontau production in the cow. Vet J. 171:500-503.

Milvae R.A. 2000. Inter-relationships between endothelin and prostaglandin $F_{2 \alpha}$ in corpus luteum function. Rev. Reprod. 5:1-5.

Milvae R.A., Hinckley S.T. \& Carlon J.C. 1996. Luteotropic and luteolytic mechanisms in the bovine corpus luteum. Theriogenology 45:1327-1349.

Miyamoto A. 1996. Intraluteal mechanisms involved in prostaglandin $\mathrm{F}_{2 \alpha}$ induced luteolysis in Ewes. J. Reprod. Dev. 42:61-63.

Morgan G., Wooding F.B. \& Godkin J.D. 1993. Localization of bovine trophoblast protein-1 in the cow blastocyst during implantation: an immunological cryoultrastructural study. Placenta 14:641-649.

Moura C.E.B., Campos D.B., Pereira F.T.V., Oliveira M.F., Miglino M.A. \& Papa P.C. 2003. Microvascularization and capillary density of corpus luteum from superovulated and control buffalo cows. Acta Microscopica 12:83-84.

Needle E., Piparo K., Cole D., Worrall C., Whitehead I., Maron G. \& Goldsmith L.T. 2007. Protein kinase A-independent cAMP stimulation of progesterone in luteal cell model is tyrosine kinase dependent but phosphatidylinositol3-kinase and mitogen-activated protein kinase independent. Biol. Reprod. March 28. [Epub ahead of print]

Nicklin L.T., Robinson R.S., Marsters P., Campbell B.K., Mann G.E. \& Hunter M.G. 2007. Leptin in the bovine corpus luteum: receptor expression and effects on progesterone production. Mol. Reprod. Dev. 74:724-729.

Niswender G.D. 2002. Molecular control of luteal secretion of progesterone. Reproduction 123:333-339.

Niswender K.D., Braden T.D. \& Niswender G.D. 1990. Nuclear changes in ovine luteal cells in response to PGF2á. Domest. Anim. Endocrinol. 7:229238.

Niswender G.D., Juengel J.L., McGuire W.J., Belfiore C.J. \& Wiltbank M.C. 1994. Luteal function: the estrous cycle and early pregnancy. Biol. Reprod. 50:239247.

Niswender G.D., Juengel J.L., Silva P.J., Rollynson M.K. \& McIntush E.W. 2000. Mechanisms controlling the function and life span of the corpus luteum. Physiol. Rev. 80:1-29.

Northey D.L. \& French L.R. 1980. Effect of embryo removal and intrauterine infusion of embryonic homogenates on the lifespan of the bovine corpus luteum. J. Anim. Sci. 50:298-302.

Ohtani M., Kobayashi S., Miyamoto A., Hayashi K. \& Fukui Y. 1998. Real-time relationships between intraluteal and plasma concentrations of endothelin, oxytocin, and progesterone during prostaglandin $\mathrm{F}_{2 \alpha}$ - induced luteolysis in the cow. Biol. Reprod. 58:103-108.

Papa P.C., Moura C.E.B., Artoni L.P., Fátima L.A., Campos D.B., Marques Jr. J.E.B., Baruselli P.S., Binelli M., Pfarrer C. \& Leiser R. 2006. VEGF-system expression in different stages of estrous cycle in superovulated and non-treated water buffalo. Domest. Anim. Endocrinol. Sept. 18. [Epub ahead of print]

Pate J.L. 1994. Cellular components involved in luteolysis. J. Anim. Sci. 72:1884-1890.

Pitzel L., Probst I., Jarry H. \& Wuttke W. 1988. Inhibitory effect of oxytocin and vasopressin on steroid release by cultured porcine luteal cells. Endocrinology 122:1780-1785.

Roberts R.M., Cross J.C. \& Leaman D.W. 1992. Interferons as hormones of pregnancy. Endocr. Rev. 13:432-452.

Schams D. \& Berisha B. 2002. Steroids as local regulators of ovarian activity in domestic animals. Domest. Anim. Endocrinol. 23:53-65.

Schams D. \& Berisha B. 2004. Regulation of Corpus luteum function in cattle: an overview. Reprod. Domest. Anim. 39:241-251.

Shaha C. \& Greenwald G.S. 1982. In vivo and In vitro production of progestins by the Corpus luteum of pregnancy of the Hamster. Biol. Reprod. 26:854-860.

Shemesh M., Hansel W. \& Strauss Iii J.F. 1984. Calcium-dependent cyclic nucleotide-independent steroidogenesis in the bovine placenta. Proc. Natl Acad. Sci. USA 81:6403-6407.

Shemesh M., Harel-Markowitz E., Gurevich M. \& Shore L.S. 1994. Staurosporine stimulates progesterone production by bovine placental cells. Biol. Reprod. 51:146-151.

Shemesh M., Izhar M., Pasmanik M. \& Shore L.S. 1992. Regulation of steroidogenesis in the bovine placenta. J. Physiol. Pharmacol. 43(Suppl.1):153-163.

Sloss V. \& Dufty J.H. 1980. Pregnancy and gestation. Handbook of Bovine Obstetrics. Williams and Wilkins, Baltimore, p.30-68.

Tsai S. \& Wiltbank M.C. 1997. ProstaglandinF2á induces expression of prostaglandin G/H Synthase-2 in the ovine corpus luteum: a potential positive feedback loop during luteolysis. Biol. Reprod. 57:1016-1022. 
Ushizawa K., Herath C.B., Kaneyama K., Shiojima S., Hirasawa A., Takahashi T., Imai K., Oshiai K., Tokunaka T., Tsunoda Y., Tsujimoto G. \& Hashizume K. 2004. cDNA microarray analysis of bovine embryo gene expression profiles during the pre-implantation period. Reprod. Biol. Endocrinol. 2:77.

Voss A.K. \& Fortune J.E. 1993. Levels of messenger ribonucleic acid for cytochrome $\mathrm{P} 450$ 17a-hydroxylase and $\mathrm{P} 450$ aromatase in preovulatory bovine follicles decrease after the luteinizing hormone surge. Endocrinology 132:2239-2245.

Wiltbank M.C., Shiao T.F., Bergfelt D.R. \& Ginther O.J. 1995. Prostaglandin F2a receptors in the early bovine corpus luteum. Biol. Reprod. 52:74-78.

Wolfenson D., Sonego H., Bloch A., Shalam-Albalancy A., Kaim M., Folman Y. $\&$ Meidan R. 2002. Seasonal differences in progesterone production by luteinized bovine thecal and granulosa cells. Domest. Anim. Endocrinol. 22:81-90.

Xie S., Low B.G., Nagel R.J., Beckers J.F. \& Roberts R.M. 1994. A novel glycoprotein of the aspartic proteinase gene family expressed in bovine placental trophectoderm. Biol. Reprod. 51:1145-1153.

Xie S., Green J., Bixby J.B., Szafranska B., DeMartini J.C., Hecht S. \& Roberts R.M. 1997. The diversity and evolutionary relationships of the pregnancyassociated glycoproteins, an aspartic proteinase subfamily consisting of many trophoblast-expressed genes. Proc. Natl Acad. Sci. USA 94:12809-12816.

Yuan W., Connor M.L. \& Buh M.M. 1993. Responsiveness of porcine large and small luteal cells to luteotropic or luteolytic hormones and cell morphologic changes during the estrous cycle and pregnancy. J. Anim. Sci. 71:481-491. 